\title{
Biomarkers for Huntington's disease: A brief overview
}

Annie Killoran ${ }^{*}$ and Kevin Biglan ${ }^{2}$

'Department of Neurology, University of lowa, IA, USA

2Department of Neurology, University of Rochester, New York, USA

\section{Article Info}

\section{Article Notes}

Received: August 02, 2016

Accepted: September 19, 2016

\section{*Correspondence:}

Dr. Annie Killoran, Department of Neurology, University of lowa, IA, USA

E-mail: annie-killoran@uiowa.edu

(c) 2016 Annie Killoran. This article is distributed under the terms of the Creative Commons Attribution 4.0 International License.

\section{Keywords}

Huntington's disease (HD)

Biomarkers

Preventative clinical trials

Pre-manifest

Neuroprotection

Huntingtin protein

Disease onset

\section{ABSTRACT}

Huntington's disease (HD) causes progressive neurological deterioration that leads to death. It is inherited in an autosomal dominant fashion, and individuals with a positive family history can be tested for the presence of the HD mutation prior to the development of the overt features that subjectively define disease onset. An objective biomarker denoting this time point would improve onset accuracy, and ideally be sufficiently sensitive to monitor progression leading up to this juncture. Once manifestations arise, patients are treated symptomatically. There are no disease-modifying treatments available for HD, but many are in development. A major goal is to develop a therapy that will delay the onset of the disease or to potentially even prevent the disease from occurring altogether. However, how does one assess the efficacy of these experimental therapeutics in individuals who carry the HD gene mutation, but are clinically-unaffected? Sensitive and reliable outcome measures are required for preventative clinical trials. Candidate biomarkers include subtle, but quantifiable abnormalities detected on clinical exam, findings on brain imaging, and levels of pathologically-relevant molecules collected in bodily fluids.

\section{Introduction}

Huntington's disease (HD) is an autosomal dominant, neurodegenerative disorder, typically presenting in young middleage. It is characterized by prominent psychiatric problems, as well as progressive deterioration in both cognitive function and motor control, leading to an early death. HD is caused by an expanded trinucleotide cytosine-adenine-guanine (CAG) repeat in the huntingtin gene ${ }^{1}$. The knowledge of this gene mutation has resulted in HD being one of the rare neurodegenerative conditions for which predictive genetic testing is available for individuals with a known family history. This enables identification of HD gene mutation carriers while they are still healthy, before developing overt clinical features of the disease. These 'pre-manifest' individuals are uniquely poised to potentially benefit from neuroprotective therapy, which would delay (or even prevent) the development of the disease's clinical manifestations and functional disability. Currently no such disease-modifying therapies are available, and HD is managed with limited, only partially-effective, symptomatic treatments. However, with the growing knowledge of the underlying pathobiology of HD, new therapies are being developed.

\section{Biomarkers}

Therapeutic clinical trials aiming to evaluate the efficacy of potential disease-modifying treatments during pre-manifest HD requires biomarkers to serve as outcome measures. Suitable 'pharmacological' or 'efficacy' biomarkers' are those that reliably and objectively respond to treatment in a predictable manner. 
Some efficacy biomarkers may also function as 'state biomarkers' or 'biomarkers of progression', which are used as indications of disease severity. Ideally, these state biomarkers reflect the underlying disease pathobiology and linearly track clinical progression throughout the disease (including during the pre-manifest stage). Though cross-sectional studies are helpful in identifying potential markers whose levels correspond to disease stage, the relationship between these candidate markers and disease progression is best evaluated by tracking results from longitudinal studies ${ }^{2}$. All biomarkers should be affordable, easily accessible, unaffected by comorbidities, and have limited variability amongst the general population ${ }^{3}$. In addition, biomarker sampling and testing should be standardized to minimize variation between facilities ${ }^{4}$. Table 1 includes candidate state biomarkers that have shown an association with stages of disease severity in both pre-manifest \& manifest HD in humans.

\section{The UHDRS and disease onset}

Currently, the United Huntington's Disease Rating Scale (UHDRS) is used to assess for treatment response in therapeutic trials in HD, as well as to monitor disease progression. It is a collection of scales that was designed to detect clinical changes in manifest HD. As such, the UHDRS may not be sensitive enough to detect the subtle features seen in some pre-manifest individuals, particularly those who are several years away from developing the disease $\mathrm{e}^{5}$. The UHDRS includes Diagnostic Confidence Level Criteria, which are used in trials to denote the clinical onset of HD. This dichotomous time point occurs when there are sufficient motor abnormalities present on physical examination, such that the examiner is $99 \%$ confident that the individual has HD. However, the interrater reliability amongst clinicians is considered only adequate, (with a weighted kappa $=0.67$, $\mathrm{SE}=0.09)^{6}$. In addition, HD patients' motor features are not a reliably constant measure, e.g. they typically worsen with stress. The subjective designation of disease onset can also seem somewhat arbitrary, given that the disease has a lengthy prodrome that includes various subtle motor and cognitive abnormalities that develop insidiously over several years ${ }^{7,8}$. The use of disease onset as an end point in clinical trials would therefore require following a large pre-manifest study population for many years, making it somewhat unrealistic ${ }^{7,8}$. An objectively measured state biomarker is needed to replace this subjective, motorcentric time-point, as well as for monitoring the severity of disease in pre-manifest individuals. Currently, pre-manifest trial subjects are staged according to the predicted duration of time until their expected disease onset; this is calculated with a non-standardized estimation using a patient's age and CAG repeat length.

\section{Clinical biomarkers: continuous measures}

Rather than relying on the UHDRS's dichotomous notion of 'disease onset', some researchers have proposed the use of continuous measures ${ }^{9}$, such as clinical features. Some UHDRS motor abnormalities can be objectively quantified, thereby improving accuracy and reducing inter-rater and intra-rater variability. For example, a modular force transducer arrangement can be used to quantify finger tapping precision, (digitomotography) ${ }^{10}$. Deficits in this task are evident even in pre-manifest HD and worsen over time, correlating with other clinical features ${ }^{11,12}$. Subtle cognitive impairment can also be detected in pre-manifest subjects. One of the more reliable measures has been the Symbol Digit Modalities test ${ }^{8,13}$, which assesses visual attention and psychomotor speed. However, such clinical markers have not proven sufficient sensitivity to subtle changes over time during the pre-manifest period ${ }^{14,15}$, so their value as outcome measures for use in preventative clinical trials is limited. In addition to this ceiling effect in pre-manifest HD, clinical biomarkers are also susceptible to floor effects in advanced disease. Plus, they can vary according to subjects' state of being, and may be confounded by the retest effect, education level and potential language barriers in non-

\begin{tabular}{|c|c|c|c|}
\hline Biomarker Source & Feature & Test & Comments \\
\hline \multirow[t]{3}{*}{ Clinical } & \multirow[t]{2}{*}{ Motor deficit } & Anti-saccade error rate ${ }^{10}$ & $\begin{array}{l}\text { Cross-sectional study; correlated with disease severity according to } \\
4 \text { groups (early and late pre-manifest and } 2 \text { groups of early (stage } 1 \\
\text { and 2) HD; early pre-manifest did not differ from controls }\end{array}$ \\
\hline & & Digitomotography ${ }^{8}$ & \multirow{2}{*}{ Large cohort; longitudinal study (36 months) } \\
\hline & Cognitive deficit & $\mathrm{SDMT}^{8}$ & \\
\hline \multirow{2}{*}{ Imaging } & Structural loss & $\mathrm{MRI}^{8}$ & Large cohort; longitudinal (36 months) \\
\hline & PDE10 uptake & [18F]MNI-659 PET $^{45}$ & Small cross-sectional study ( 8 early HD and 3 pre-manifest HD) \\
\hline \multirow{3}{*}{ Wet: blood } & \multirow{2}{*}{ Immune system } & $\mathrm{IL}-8^{46}$ & \multirow{2}{*}{$\begin{array}{l}\text { Cross-sectional study; correlated with disease severity according to } 3 \\
\text { groups (pre-manifest, early and moderate HD) }\end{array}$} \\
\hline & & TNF- $\alpha^{46}$ & \\
\hline & Neuro-degeneration & $\mathrm{NfL}^{47}$ & Only 3 pre-manifest included; not HD-specific \\
\hline
\end{tabular}

Abbreviations: HD, Huntington's Disease; SDMT, Symbol Digit Modalities test; MRI, Magnetic resonance imaging; PDE10, phosphodiesterase 10A; [18F]MNI-659 PET, 2-(2-(3-(4-(2-[18F]fluoroethoxy)phenyl)-7-methyl-4-oxo-3,4-dihydroquinazolin-2-yl)ethyl)-4-isopropoxyisoindoline1,3-dione positron emission tomography; IL-8, Interleukin 8; TNF- $\alpha$, Tumor Necrosis Factor-alpha; NfL, neurofilament light chain

Table 1: Summary of candidate HD state biomarkers which associated with disease severity in pre-manifest and manifest HD. 
native speakers ${ }^{3}$. Finally, relying on clinical biomarkers has the added challenge of trying to distinguish between a treatment's symptomatic improvement vs underlying disease modification ${ }^{3}$.

\section{Imaging biomarkers}

In HD trials, clinical outcome measures are typically used in combination with brain imaging, which has proven to be a more robust marker during the pre-manifest period ${ }^{14}$. For example, in the PRECREST study, the rate of cortical thinning was significantly reduced at 6 and 18 months in subjects who were at risk for HD on creatine treatment ${ }^{14}$. This is despite there being no detectable clinical change in the study subjects, which is not unexpected given the scarcity of clinical examination findings at this prodromal stage of disease. Ultimately one would want to validate the imaging findings against the cohort's future clinical outcomes. Using brain atrophy as an efficacy measure can be limited by the typically slow rate at which it occurs, making it somewhat impractical for most clinical trials. Compared to structural imaging, functional and metabolic imaging techniques may be more sensitive to change over short periods of time. These techniques may also allow for some interesting visualization of potentially-reversible findings that match nicely with clinical features ${ }^{2}$. For example, compared to riluzole-treated HD subjects, those treated with placebo had reduced metabolic FDG uptake in the parietal lobe that linearly correlated with worsening motor scores. Whereas reduced uptake in the frontal and temporal areas correlated with worsening behavioral measures ${ }^{16}$.

Structural imaging has shown utility in monitoring disease progression in the very early pre-manifest stage. Volume loss, notably in the striatum, is detectable 1-2 decades prior to the development of motor features ${ }^{17-19}$. The atrophy progresses over time ${ }^{18}$ and correlates with disease load ${ }^{20}$. This supports the use of structural imaging as a state biomarker, though it is limited in that it does not provide a direct pathological measure of disease.

\section{Wet biomarkers:}

Wet biomarkers, (those obtained from bodily fluids), are another potential source of useful outcome measures, particularly if they reflect the disease's pathobiology. Various pathologic mechanisms have been implicated in $\mathrm{HD}$, yielding numerous potential molecular markers.

For example, the pathogenesis of HD seems to involve the dysregulation of gene transcription through posttranslational modifications of histones. H2AFY is a particular histone that is elevated in individuals with $\mathrm{HD}^{21}$, though it does not track disease progression, making it unsuitable as a state biomarker. However, it has shown promise as a potential therapeutic marker. In subjects with early HD, H2AFY levels responded to treatment with
Sodium Phenylbutyrate, a histone deacetylase (HDAC) inhibitor, which is known to suppress neurodegeneration in mouse models of $\mathrm{HD}^{21}$.

8-hydroxy-2-deoxyguanosine $(8-\mathrm{OHdG})$ is a measure of oxidative stress ${ }^{22}$, which is believed to be involved in the pathogenesis of $\mathrm{HD}^{23}$. As a hopeful candidate biomarker ${ }^{24}$, it was the focus of numerous studies. Elevated 8-OHdG was detected in HD subjects' brain and blood in several studies $^{25-29}$, (though not all) ${ }^{30}$, and levels correlated with proximity to projected disease onset ${ }^{31}$. Unfortunately, larger investigations found no association with disease severity ${ }^{10,32}$, making it unsuitable as a state biomarker. However, 8-OHdG also showed promise as an efficacy biomarker in antioxidant trials ${ }^{33,34}$, including one in a murine model of $\mathrm{HD}$, in which there was significant improvement $^{38}$. In human HD studies, 8-OHdG levels also decreased following treatment with the antioxidants creatine $^{27}$, and Coenzyme $\mathrm{Q} 10(\mathrm{CoQ})^{30}$, but it remained unchanged in a subsequent, pre-manifest trial of $\mathrm{CoQ}^{38}$, refuting its reliability. 8-OHdG also lacked HD-specificity, which is a common short-coming amongst candidate biomarkers. For example, serum titers of the inflammatory marker angiotensin II type 1 receptors (AT1R) antibody correlated with the UHDRS motor score, as well as with smoking and concurrent infection, thus making results potentially misleading ${ }^{37}$.

One potential wet biomarker being studied that is presumably HD-specific is the mutant Huntingtin protein (mHtt). This is an attractive candidate given that it is the principal pathogenic molecule of HD. In addition, besides being detected in $\mathrm{CSF}^{38,39}$ and blood ${ }^{40}$, it is also measurable in saliva ${ }^{41}$, providing convenient, non-invasive access to a protein whose damage is focused on the central nervous system. In HD gene carriers, peripheral immune cell levels of mHTT are elevated, with differences in mean mHTT between pre-manifest and early-stage HD subjects, but not between early-stage and moderate-stage subjects ${ }^{40}$. Levels of $\mathrm{mHtt}$ also associate with disease burden scores and caudate atrophy rates in gene positive individuals ${ }^{40}$. One study found CSF mHtt levels associated with motor and cognitive features in pre-manifest and early-mid HD, but not in late $\mathrm{HD}^{38}$. However, an earlier study demonstrated its correlation with UHDRS motor scores in individuals at stages ranging from pre-manifest to advanced disease ${ }^{42}$.

MHtt has yet to be tried as an efficacy biomarker in humans, but in animal studies it has shown utility as a pharmacologic marker of nucleotide-based gene silencing therapies aimed at lowering mHtt. Both antisense oligonucleotides (ASO) and RNA interference (RNAi) reduced levels of $\mathrm{mHtt}$ in the striatum and CSF, which in turn corresponded with significant phenotypic improvements $^{43,44}$. In humans, the first gene silencing trial in HD is currently underway, (NCT02519036). It 
is evaluating an antisense oligonucleotide that targets mutant Huntingtin RNA, which is expected to result in reduced $\mathrm{mHtt}$ production and therefore less of the neuronal destruction that is characteristic of HD. Naturally, one of the study's outcome measures is $\mathrm{mHtt}$, functioning as an efficacy biomarker.

\section{Conclusion}

The availability of predictive genetic testing has enabled for large observational trials of pre-manifest individuals for the characterization of their insidiously developing clinical features. Subjects are staged by the amount of time that is predicted for them to reach the subjective and motoricallydefined disease onset. A reliable state biomarker (or set of biomarkers) that is sufficiently sensitive for use in the pre-manifest stage is needed for marking progression during this early part of the disease, as well as for a more accurately defined 'onset'.

Predictive genetic testing has also made HD the ideal disorder for which to develop a neuroprotective treatment, with the goal of delaying or even preventing the clinical features of the disease. The ability to assess the therapeutic response to experimental drugs is a major challenge at the pre-manifest stage. Research efforts are currently geared towards finding reliable and objective biomarkers to suit this purpose. A range of clinical abnormalities are detectable in pre-manifest HD, but with limited sensitivity to change over time. Wet biomarkers that reflect the pathobiology of HD are logical candidates being explored. Current hope lies in pathologically-relevant molecules such as $\mathrm{mHtt}$, most likely in combination with other quantifiable markers, notably measurable changes detected on brain imaging.

\section{Funding}

Kevin Biglan has served as a consultant for KJT and UCB and has received research funding from AMC Health, Michael J. Fox Foundation, National Institute of Neurological Disorders and Stroke, National Parkinson Foundation, Lundbeck, Huntington's Disease Society of America, Patient-Centered Outcomes Research Institute, and Greater Rochester Health Foundation.

\section{References}

1. The Huntington's Disease Collaborative Research Group. A novel gene containing a trinucleotide repeat that is expanded and unstable on Huntington's disease chromosomes. Cell. 1993;72(6):971-983.

2. Weir DW, Sturrock A, Leavitt BR. Development of biomarkers for Huntington's disease. The Lancet Neurology. 2011;10(6):573-590.

3. Henley SM, Bates GP, Tabrizi SJ. Biomarkers for neurodegenerative diseases. Current Opinion in Neurology. 2005;18:698-705.

4. Byrne LM, Wild EJ. Cerebrospinal fluid Biomarkers for Huntington's disease. Journal of Huntington's Disease. 2016;5(1):1-13.

5. Paulsen JS, Wang $\mathrm{C}$, Duff $\mathrm{K}$, et al. Challenges assessing clinical endpoints in early Huntington disease. Movement Disorders. 2010;25(15):2595-2603.
6. 6 Hogarth P, Kayson E, Kieburtz K, Marder K, Oakes D, Rosas D. Interrater agreement in the assessment of motor manifestations of Huntington's disease. Movement Disorders. 2005;20(3):293-297.

7. Huntington Study Group PHAROS Investigators. At risk for Huntington disease: the PHAROS (Prospective Huntington at Risk Observational Study) cohort enrolled. Archives of Neurology. 2006;63(7):991-996.

8. Tabrizi SJ, Scahill RI, Owen G, et al. TRACK-HD Investigators. Predictors of phenotypic progression and disease onset in premanifest and early-stage Huntington's disease in the Track-HD study: analysis of 36-month observational data. Lancet Neurology. 2013;12(7):637649.

9. Biglan KM, Shoulson I, Kieburtz K, et al. Clinical-genetic associations in the prospective Huntington at risk observational study (PHAROS). JAMA Neurology. 2016;73(1):102.

10. Tabrizi, SJ, Langbehn, DR, Leavitt, BR et al. Biological and clinical manifestations of Huntington's disease in the longitudinal TRACKHD study: cross-sectional analysis of baseline data. Lancet Neurology. 2009;8:791-801.

11. Tabrizi SJ, Reilmann R, Roos RA, et al. Potential endpoints for clinical trials in premanifest and early Huntington's disease in the TRACK-HD study: Analysis of 24 month observational data. Lancet Neurology. 2012;11(1):42-53.

12. Bechtel N, Scahill RI, Rosas HD, et al. Tapping linked to function and structure in premanifest and symptomatic Huntington disease. Neurology. 2010;75(24):2150-2160.

13. Paulsen JS, Smith MM, Long JD; PREDICT HD investigators and Coordinators of the Huntington Study Group. Cognitive decline in prodromal Huntington disease: implications for clinical trials. J Neurol Neurosurg Psychiatry. 2013;84(11):1233-1239.

14. Rosas HD, Doros G, Gevorkian S, et al. PRECREST: A phase II prevention and biomarker trial of creatine in at-risk Huntington disease. Neurology. 2014;82(10):850-857.

15. Stout JC, Jones R, Labuschagne I, et al. Evaluation of longitudinal 12 and 24 month cognitive outcomes in premanifest and early Huntington's disease. Journal of Neurology, Neurosurgery \& Psychiatry. 2012;83:687-694.

16. Squitieri F, Orobello S, Cannella M, et al. Riluzole protects Huntington disease patients from brain glucose hypometabolism and grey matter volume loss and increases production of neurotrophins. European Journal of Nuclear Medicine and Molecular Imaging. 2009;36:111320.

17. Tabrizi SJ, Scahill RI, Durr A, et al. Biological and clinical changes in premanifest and early stage Huntington's disease in the TRACKHD study: The 12-month longitudinal analysis. Lancet Neurology. 2011;10(1):31-42.

18. Paulsen JS, Langbehn DR, Stout JC, et al. Detection of Huntington's disease decades before diagnosis: The Predict-HD study. Journal of Neurology, Neurosurgery \& Psychiatry. 2008;79(8):874-880.

19. Aylward EH, Sparks BF, Field KM, et al. Onset and rate of striatal atrophy in preclinical Huntington disease. Neurology. 2004;63(1):6672.

20. van Oostrom JCH, Maguire RP, Verschuuren-Bemelmans CC, et al. Striatal dopamine D2 receptors, metabolism, and volume in preclinical Huntington disease. Neurology. 2005;65(6):941-943.

21. Hu Y, Chopra V, Chopra R, et al. Transcriptional modulator H2A histone family, member Y (H2AFY) marks Huntington disease activity in man and mouse. Proceedings of the National Academy of Sciences of the United States of America. 2011;108:17141-17146.

22. Hermans N, Cos P, Maes L, et al. Challenges and pitfalls in Antioxidant research. Current Medicinal Chemistry. 2007;14(4):417-430.

23. Reddy PH, Mao P, Manczak M. Mitochondrial structural and functional 
dynamics in Huntington's disease. Brain Research Reviews. 2009;61(1):33-48.

24. Killoran A, Biglan KM. 8-OHdG: Its (limited) potential as a biomarker for Huntington's disease. Biomarkers in Medicine. 2012;6(6):777780 .

25. Browne SE, Bowling AC, Macgarvey U, et al. Oxidative damage and metabolic dysfunction in Huntington's disease: Selective vulnerability of the basal ganglia. Annals of Neurology. 1997;41(5):646-653.

26. Polidori MC, Mecocci P, Browne SE, Senin U, Beal MF. Oxidative damage to mitochondrial DNA in Huntington's disease parietal cortex. Neuroscience Letters. 1999;272(1):53-56.

27. Hersch SM, Gevorkian S, Marder K, et al. Creatine in Huntington disease is safe, tolerable, bioavailable in brain and reduces serum 80H2'dG. Neurology. 2006;66(2):250-252.

28. Túnez I, Sánchez-López F, Agüera E, et al.: Important role of oxidative stress biomarkers in Huntington's disease. Journal of Medicinal Chemistry. 2011;54(15):5602-6

29. Chen C-M, Wu Y-R, Cheng M-L, et al. Increased oxidative damage and mitochondrial abnormalities in the peripheral blood of Huntington's disease patients. Biochemical and Biophysical Research Communications. 2007;359(2):335-340.

30. Biglan KM, Dorsey ER, Evans RV, et al. Plasma 8-hydroxy-2 -deoxyguanosine Levels in Huntington Disease and Healthy Controls Treated with Coenzyme Q10. Journal of Huntington's Disease. 2012;1(1):65-69.

31. Long JD, Matson WR, Juhl AR, Leavitt BR, Paulsen JS. 80HdG as a marker for Huntington disease progression. Neurobiology of Disease. 2012;46(3):625-634.

32. Borowsky B, Warner J, Matson W, et al. $80 \mathrm{HdG}$ is not a biomarker for Huntington's disease; lessons for future biomarker studies. [EHDN abstract F14]. Journal of Neurology, Neurosurgery \& Psychiatry. 2012;83(Suppl 1):A25-A26.

33. Boesch S, Sturm B, Hering S, et al. Neurological effects of recombinant human erythropoietin in Friedreich's ataxia: A clinical pilot trial. Movement Disorders. 2008;23(13):1940-1944.

34. Bowen P, Chen L, Stacewicz-Sapuntzakis M, et al.: Tomato sauce supplementation and prostate cancer: lycopene accumulation and modulation of biomarkers of carcinogenesis. Experimental Biology and Medicine. 2002;227(10):886-93.

35. Smith KM, Matson S, Matson WR, et al. Dose ranging and efficacy study of high-dose coenzyme Q10 formulations in Huntington's disease mice. Biochimica et Biophysica Acta. 2006 Jun;1762(6):616-26.

36. Biglan KM, Ross CA, Killoran A, et al. for the Huntington Study Group PREQUEL Investigators. 80HdG levels in response to Coenzyme
Q10 in the PREQUEL study of pre-manifest Huntington disease. [MDS International Congress late-breaking abstract-40]. Movement Disorders. 2013.

37. Lee D-H, Heidecke H, Schröder A, et al. Increase of angiotensin II type 1 receptor auto-antibodies in Huntington's disease. Molecular Neurodegeneration. 2014;9:49.

38. Wild EJ, Boggio R, Langbehn D, et al. Quantification of mutant huntingtin protein in cerebrospinal fluid from Huntington's disease patients. Journal of Clinical Investigation. 2015;125(5):1979-1986.

39. Southwell AL, Smith SEP, Davis TR, et al. Ultrasensitive measurement of huntingtin protein in cerebrospinal fluid demonstrates increase with Huntington disease stage and decrease following brain huntingtin suppression. Scientific Reports. 2015;5:12166.

40. Weiss A, Trager U, Wild EJ, et al. Mutant huntingtin fragmentation in immune cells tracks Huntington's disease progression. Journal of Clinical Investigation. 2012;122:3731-3736.

41. Corey-Bloom J, Aikin A, Garza M, et al. Salivary Huntington protein as a peripheral biomarker for Huntington's disease. [AAN Annual Meeting Abstract P1.053] 2016.

42. Wild EJ, Boggio R, Langbehn D, et al. Quantification of mutant huntingtin protein in cerebrospinal fluid from Huntington's disease patients. Journal of Clinical Investigation. 2015;125(5):1979-1986.

43. Kordasiewicz HB, Stanek LM, Wancewicz EV, et al. Sustained therapeutic reversal of Huntington's disease by transient repression of Huntingtin synthesis. Neuron. 2012;74(6):1031-1044.

44. Stanek LM, Sardi SP, Mastis B, et al. Silencing mutant Huntingtin by Adeno-Associated virus-mediated RNA interference Ameliorates disease manifestations in the YAC128 mouse model of Huntington's disease. Human Gene Therapy. 2014;25(5):461-474.

45. Russell DS, Barret O, Jennings DL, et al.. The phosphodiesterase 10 positron emission tomography tracer, [18F]MNI-659, as a novel biomarker for early Huntington disease. JAMA Neurology. 2014;71:1520-1528.

46. Björkqvist M, Wild EJ, Thiele J, Silvestroni A, Andre R, et al. A novel pathogenic pathway of immune activation detectable before clinical onset in Huntington's disease. The Journal of Experimental Medicine. 2008;205:1869-1877.

47. Rodrigues FB, Byrne L, Tabrizi SJ, Zetterberg H, Wild E. CSF inflammatory and cell death biomarkers in Huntington's disease - An exploratory cross-sectional study [abstract]. Movement Disorders. 2016; 31 (suppl 2). http://www.mdsabstracts.org/abstract/csfinflammatory-and-cell-death-biomarkers-in-huntingtons-diseasean-exploratory-cross-sectional-study/. Accessed July 10, 2016. 